\title{
Anger management
}

DOI:

10.1038/nrc1985

\section{URLs}

Nodal

http://www.ncbi.nlm.nih.gov/ entrez/query.fcgi?db=gene\&c $\mathrm{md}=$ RetrieveEdopt=full_ reportElist_uids $=4838$

TGF $\beta$

http://www.ncbi.nlm.nih.gov/ entrez/query.fcgi?db=gene\&c $\mathrm{md}=$ Retrieve\&dopt=full_ report\&list_uids $=7040$
Metastatic tumour cells share many characteristics with embryonic progenitor cells, so investigating the behaviour of metastatic cells during embryogenesis in models such as zebrafish (Danio rerio) can reveal common functional aspects. Mary Hendrix and colleagues have found that aggressively metastatic melanoma cells can disrupt early embryonic tissue patterning because they secrete the morphogen Nodal. Furthermore, they show that the expression of Nodal in human melanomas correlates well with aggressive forms of the disease.

Hendrix and colleagues have previously shown that when injected into zebrafish embryos, metastatic melanoma cells can respond to many environmental cues and do not form tumours. To explore the biology of aggressive melanoma cells more comprehensively within the zebrafish system, the authors investigated whether human melanoma cells could affect early stages of zebrafish development.

The injection of aggressively metastatic melanoma cells resulted in the formation of an additional body axis. Although many signalling pathways, such as Wnt and transforming growth factor- $\beta$ (TGF $\beta$ ), can produce a similar effect, only a few factors can produce such well-formed axes. Nodal is one such factor, and the authors found that highly aggressive melanoma cells express Nodal, whereas less aggressive melanoma cells do not. In agreement with this,
Hendrix and colleagues found that Nodal expression was absent in normal human skin samples, was only weakly expressed in primary melanomas, but was expressed in $60 \%$ of metastatic melanoma samples, as determined by immunostaining.

Nodal is a member of the TGF $\beta$ superfamily and binds the activinlike (Alk) family of kinase receptors. As Nodal is known to be involved in maintaining the pluripotent status (plasticity) of embryonic stem cells, the authors examined the expression of differentiation markers in the aggressive cells. The inhibition of Alk receptors (which reduces the expression of Nodal) or the expression of Nodal-targeted oligonucleotides increased the expression of melanocyte differentiation markers in the aggressive melanoma cells, indicating that Nodal expression impedes differentiation and promotes plasticity.

Importantly, inhibiting Nodal expression also suppressed the capacity of the aggressive melanoma cells to invade the extracellular matrix, to form colonies in soft agar and to form tumours when injected subcutaneously into nude mice.

These results indicate that the expression of Nodal might be a good diagnostic marker for disease progression in patients with melanoma, and that Nodal is a potential target for the treatment of aggressive disease.

Nicola McCarthy

ORIGINAL RESEARCH PAPER Topczewska, J. M et al. Embryonic and tumorigenic pathways converge via Nodal signaling: role in melanoma aggressiveness. Nature Med. 30 July 2006 (doi:10.1038/nm1448)

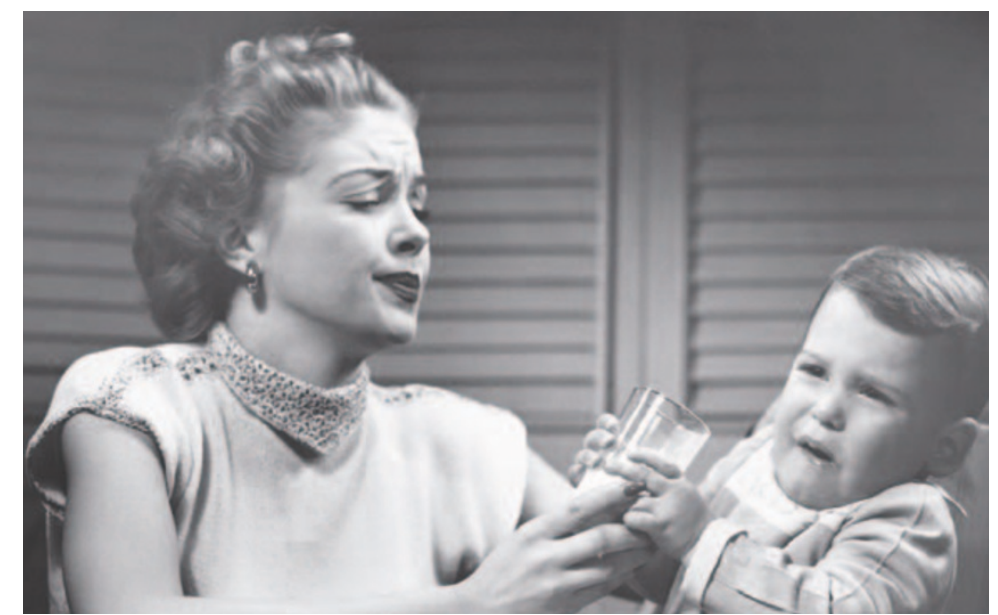

